\title{
Prediction of Aerodynamics Coefficients of Modified NACA 4415 Airfoil Using Computational Fluid Dynamics
}

\author{
Sudarsono*, Saiful Huda, and Toto Rusianto \\ Dept. Of Mechanical Engineering, Faculty of Industrial Technolofy, Institut Sains \& Teknologi \\ AKPRIND Yogyakarta, Indonesia
}

\begin{abstract}
The aim of the resent work is to predict the aerodynamic performance of modified NACA 4415 airfoil using computational fluid dynamics modelling. The performance is compared to that of NACA 4415 Standard. The simulation is performed using FLUENT with the variables of freestream velocity (i.e. $3,4,5$, and $18 \mathrm{~m} / \mathrm{s}$ ) and angle of attacks (i.e. $-12^{\circ}$, $10^{\circ},-8^{\circ},-6^{\circ}, 0^{\circ}, 6^{\circ}, 12^{\circ}, 14^{\circ}, 16^{\circ}, 18^{\circ}$, and $\left.20^{\circ}\right)$. The results indicate that the modified NACA 4415 airfoil has better lift and momentum coefficient. The value of lift coefficient of modified NACA 4415 is relatively higher than NACA 4415 , especially at $-12^{\circ}<\alpha<-8^{\circ}$ and $12^{\circ}<\alpha<20^{\circ}$. In addition, the momentum coefficient of the modified NACA 4415 is also higher than the NACA 4415 at $-12^{\circ}<\alpha<20^{\circ}$.
\end{abstract}

\section{Introduction}

Due the shortage of conventional fuel like gasoline and diesel fuel, many alternative energy sources have been explored. One of the alternative energies which is suitable utilized in Indonesia is wind energy. Indonesia has a wind energy potential about $9.29 \mathrm{GW}$, but only $0.0006 \mathrm{GW}$ of those potential has been utilized [1] through wind turbine energy conversion system.

In wind turbine energy conversion system especially small scale capacity, it is important to build the propeller which has good aerodynamics performance. The selection of the airfoil for the propeller is crucial part in propeller design and fabrication. Computational Fluid Dynamics (CFD) modelling is useful tool to predict aerodynamics performance of any type of airfoil [2-6]. The use of the CFD in design optimization is very helpful to reduce design cost and to obtain the most suitable airfoil of the propeller for particular wind velocities [79].

Oukassou et al. [10] compared the power, lift, and drag between NACA 0012 airfoil and NACA 2412. They used three models the Spalart-Allmaras, the k-epsilon (RNG) and the komega shear stress transport (SST). They obtained that the simulation results were good concordances compared with the experimental results. Simulation of airfoil S809 using two models of steady Reynolds-Averaged Navier-Stokes (RANS), and unsteady Reynolds-

* Corresponding author: sudarsono1574@akprind.ac.id 
Averaged Navier-Stokes (URANS) was conducted by Xu et al. [11]. Meanwhile, the effect of angle of attack and tip speed ratio on horizontal axis wind turbine have been performed by Wen et al. [12] and Bakiri et al. [13], respectively]. The angle of attack fluctuated under yawed conditions and could be attributed to three effects: the blade advancing \& retreating effect, the non-uniform induction effect and the upwind \& downwind yawing effect. At a positive yaw angle, the blade advancing and retreating effect results in a maximum angle of attack at the azimuth of $0^{\circ}$. Form six HWAT blades investigated by Bakiri, the optimum Tip Speed Ratio was 7.3. Meanwhile, Sudarsono et al, 2019 [14] found that maximum predicted output power of modified NACA 4415 was 6 at wind speed of $7 \mathrm{~m} / \mathrm{s}$ using three dimensional CFD analysis.

In the present work, the aerodynamic performance of airfoils NACA 4415 Standard and Modified NACA 4415 is predicted using computational fluid dynamic analysis which is performed in FLUENT software. The analysis is run at free stream velocity of $3,4,5$, and 18 $\mathrm{m} / \mathrm{s}$ and at angle of attacks of $-12^{\circ},-10^{\circ},-8^{\circ},-6^{\circ}, 0^{\circ}, 6^{\circ}, 12^{\circ}, 14^{\circ}, 16^{\circ}, 18^{\circ}$, and $20^{\circ}$.

\section{Methodology}

\subsection{Pre-processing}

Fig.1 the technical drawing of the wind turbine propeller in the present work. The propeller is designed based on NACA 4415 airfoil. The chord length is $850 \mathrm{~mm}$ and maximum width at the base is $170 \mathrm{~mm}$ and the minimum width of the tip is $75 \mathrm{~mm}$. In the pre-processing step, GAMBIT software is used to draw the airfoil model and to mesh the model and its computation domain as displayed in Fig. 2. The meshing of the computational in this simulation is 29400 meshes.

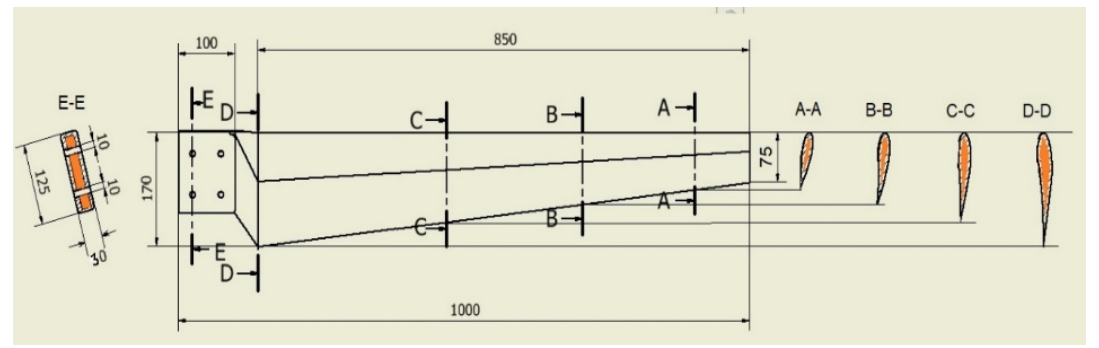

Fig. 1. Propeller of NACA 4415 and its detail dimension 


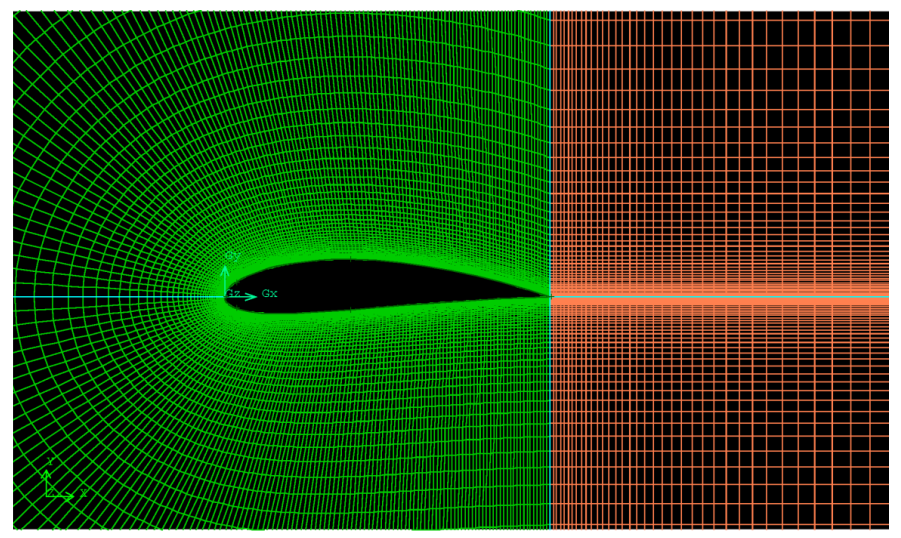

Fig. 2. The meshing of the computational domain (29400 meshes)

The next step in pre-processing is a selection of the solver, a setting of initial condition, fluid properties, and boundary condition. Simulation is run in the solver step. The solver FLUENT working procedure is shown in Fig. 3.

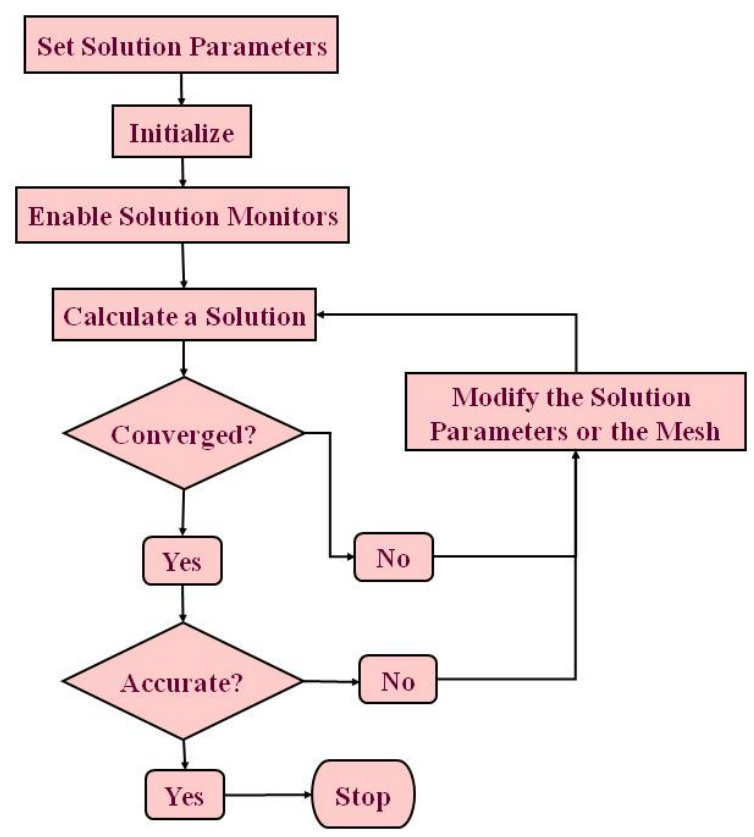

Fig. 3. Flow diagram of the solver in FLUENT [15]

\subsection{The Solver}

In the present work, the simulation is performed at freestream velocities (Uळ) of $3,4,5$, and $18 \mathrm{~m} / \mathrm{s}$ ) and angles of attack $(\alpha)$ of $-12^{\circ},-10^{\circ},-8^{\circ},-6^{\circ}, 0^{\circ}, 6^{\circ}, 12^{\circ}, 14^{\circ}, 16^{\circ}, 18^{\circ}$, and $20^{\circ}$ at those velocities, the Reynolds number are 41000, 55000, 68000, and 82000, respectively. The turbulent model of Spallart Allmaras (S-A) is used. The S-A model is very simple which only need to solve turbulent kinematic viscosity. The kinematics viscosity is quantified using Eq. (1) 


$$
\rho \frac{\mathrm{Du}}{\mathrm{Dt}}=\mathrm{G}_{\mathrm{v}}+\frac{1}{\sigma_{\bar{v}}}\left[\frac{\partial}{\partial \mathrm{x}_{\mathrm{j}}}\left\{(\mu+\rho \overline{\mathrm{v}}) \frac{\partial \overline{\mathrm{v}}}{\partial \mathrm{x}_{\mathrm{j}}}\right\}+\mathrm{C}_{\mathrm{b} 2} \rho\left(\frac{\partial \overline{\mathrm{v}}}{\partial \mathrm{x}_{\mathrm{j}}}\right)^{2}\right]-\mathrm{Y}_{\mathrm{v}}
$$

Where $G_{v i s}$ the turbulent viscosity is generated, $Y_{v}$ is the destruction of turbine viscosity, $\sigma$ and $\mathrm{C}$ is the coefficient, and $\mathrm{U}$ is the air kinematic viscosity. Aerodynamics coefficients of lift (cl), drag (cd), and momentum (cm) are solved using Eq. (2) to Eq. (9)

$$
\begin{aligned}
\mathrm{c}_{\mathrm{P}} & =\frac{\mathrm{P}-\mathrm{P}_{\infty}}{\frac{1}{2} \rho \mathrm{U}_{\infty}^{2}} \\
\mathrm{c}_{\mathrm{f}} & =\frac{\tau}{\frac{1}{2} \rho \mathrm{U}_{\infty}^{2}} \\
\mathrm{c}_{\mathrm{n}} & =\frac{1}{\mathrm{c}} \int_{0}^{\mathrm{c}}\left(\mathrm{c}_{\mathrm{P}, \text { lower }}-\mathrm{c}_{\mathrm{P}, \text { upper }}\right) \mathrm{dx}+\frac{1}{\mathrm{c}} \int_{0}^{\mathrm{c}}\left(\mathrm{c}_{\mathrm{f}, \text { upper }} \frac{\mathrm{dy} \mathrm{y}_{\text {upper }}}{\mathrm{dx}}+\mathrm{c}_{\mathrm{f}, \text { lower }} \frac{\mathrm{dy}_{\text {lower }}}{\mathrm{dx}}\right) \mathrm{dx} \\
\mathrm{c}_{\mathrm{a}} & =\frac{1}{\mathrm{c}} \int_{0}^{\mathrm{c}}\left(\mathrm{c}_{\mathrm{P}, \text { upper }} \frac{\mathrm{dy} \mathrm{y}_{\text {upper }}}{\mathrm{dx}}-\mathrm{c}_{\mathrm{P}, \text { lower }} \frac{\mathrm{dy}}{\mathrm{d} \mathrm{dx}}\right) \mathrm{dx}+\frac{1}{\mathrm{c}} \int_{0}^{\mathrm{c}}\left(\mathrm{c}_{\mathrm{f}, \text { upper }}+\mathrm{c}_{\mathrm{f}, \text { lower }}\right) \mathrm{dx} \\
c_{m} & =\frac{1}{c^{2}} \int_{0}^{c}\left(c_{P, \text { upper }}-c_{P, \text { lower }}\right) x d x-\frac{1}{c^{2}} \int_{0}^{c}\left(c_{f, \text { upper }} \frac{d y_{\text {upper }}}{d x}+c_{f, \text { lower }} \frac{d y_{\text {lower }}}{d x}\right) x d x \\
\frac{1}{c^{2}} \int_{0}^{c}\left(c_{P, \text { upper }} \frac{d y_{\text {upper }}}{d x}+c_{f, \text { upper }}\right) y_{\text {upper }} & d x+\frac{1}{c^{2}} \int_{0}^{c}\left(c_{f, \text { lower }}-c_{P, \text { lower }} \frac{d y_{\text {lower }}}{d x}\right) y_{\text {lower }} d x \\
\mathrm{c}_{\mathrm{m}, 1 / 4 \text { chord }}=\mathrm{c}_{\mathrm{m}}-\frac{1}{4} \mathrm{c}_{1} & \mathrm{c}_{\mathrm{d}}=
\end{aligned}
$$

\section{Results and Discussion}

Fig. 4 shows the value of $y+$ in front the airfoil for the freestream velocity of $18 \mathrm{~m} / \mathrm{s}$ and angle of attack $(\alpha)$ of $20^{\circ}$. The $y+$ lies between 4.2 and 21.8 which indicates that the transition flow occurs near the wall due to viscous and inertia forces. 


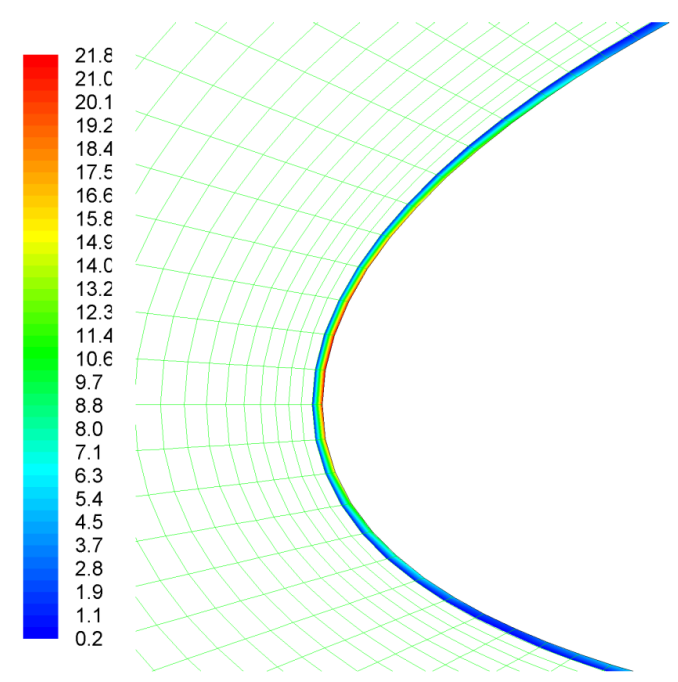

Fig. 4. The value of $y+$ in front of the airfoil

Meanwhile, the contours of the freestream near the wall at various angles of attack are shown in Fig. 5. The Figure indicates that at $\alpha$ of -12 , the flow towards the lower right direction in the direction of the freestream air velocity flow and a flow vortex formed subsurface airfoil with low flow velocity (in blue) with a range of $0-0.65 \mathrm{~m} / \mathrm{s}$. Vortex flow will disappear gradually with increasing $\alpha$ to the value of $0^{\circ}$ as shown in the Fig. 5 for $\alpha$ of $10^{\circ},-8^{\circ},-6^{\circ}$ and $0^{\circ}$. As a result the value of lift force has a negative value or lift force downward and lift force gradually goes upward with the increasing value of the angle of attack from $-12^{\circ}$ degrees to $0^{\circ}$ degrees. The vortex air will begin to occur in the upper surface of the airfoil gradually with increasing $\alpha$ from $0^{\circ}$ to $20^{\circ}$. As a result, the lift force increases to the top and reaches a maximum value at $\alpha$ of $14^{\circ}$ and the lift value starts to decrease at $\alpha$ of $16^{\circ}, 18^{\circ}$ and $20^{\circ}$. The same phenomenon will occur for other Uø (i.e. 4,5 and $18 \mathrm{~m} / \mathrm{s}$ ).

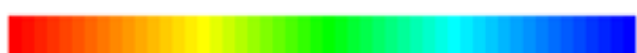

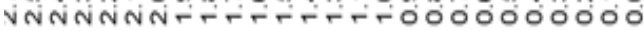

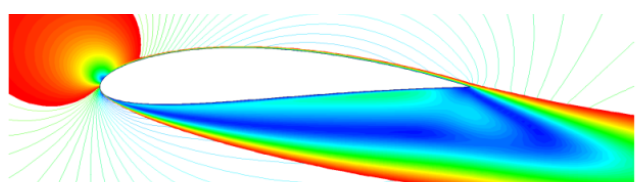

$\alpha=-12^{\circ}$

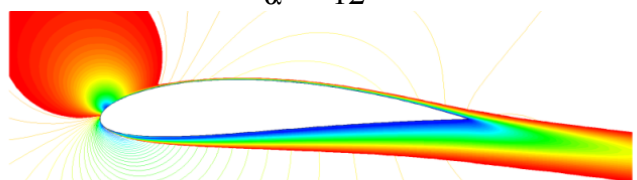

$$
\alpha=-10^{\circ}
$$

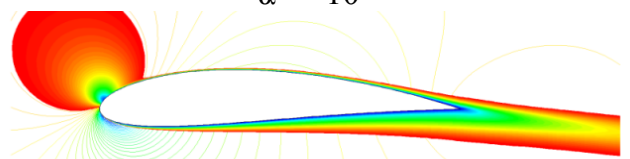

$\alpha=-8^{\circ}$
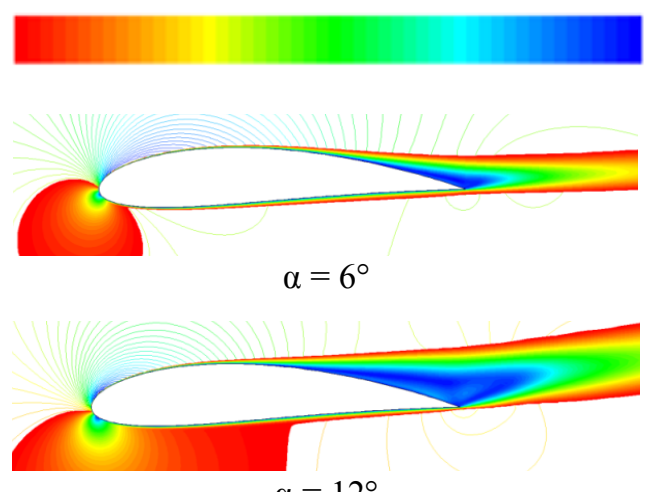

$\alpha=12^{\circ}$

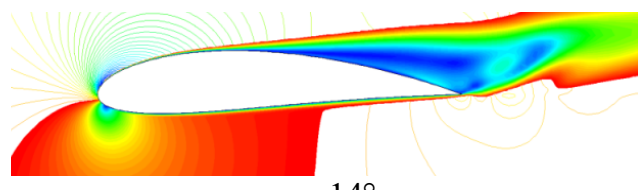

$\alpha=14^{\circ}$ 


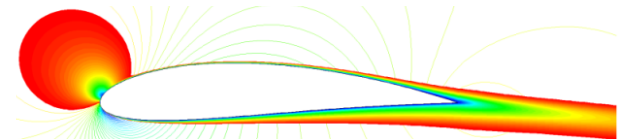

$\alpha=-6^{\circ}$

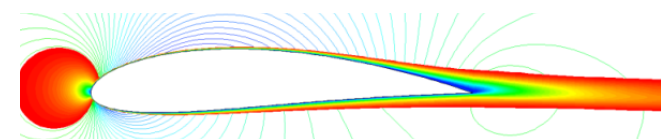

$\alpha=0^{\circ}$



$\alpha=16^{\circ}$

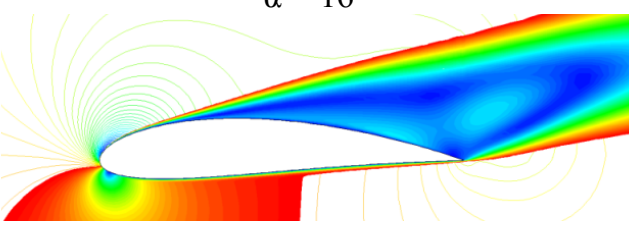

$\alpha=18^{\circ}$

Fig. 5. The contours of the flow at $\mathrm{U}_{\infty}=3 \mathrm{~m} / \mathrm{s}$ and various angles of attack $(\alpha)$

Fig. 6 displays the coefficient of aerodynamic of the airfoils NACA 4415 and modified NACA 4415 at Reynold numbers of 41000, 55000, 68000, and 82000. Based on the graph, it appears that the lift coefficient value of NACA 4415 is relatively greater than NACA 4415 , especially at angle of attack $-12^{\circ}<\alpha<-8^{\circ}$ and $12^{\circ}<\alpha<20^{\circ}$.
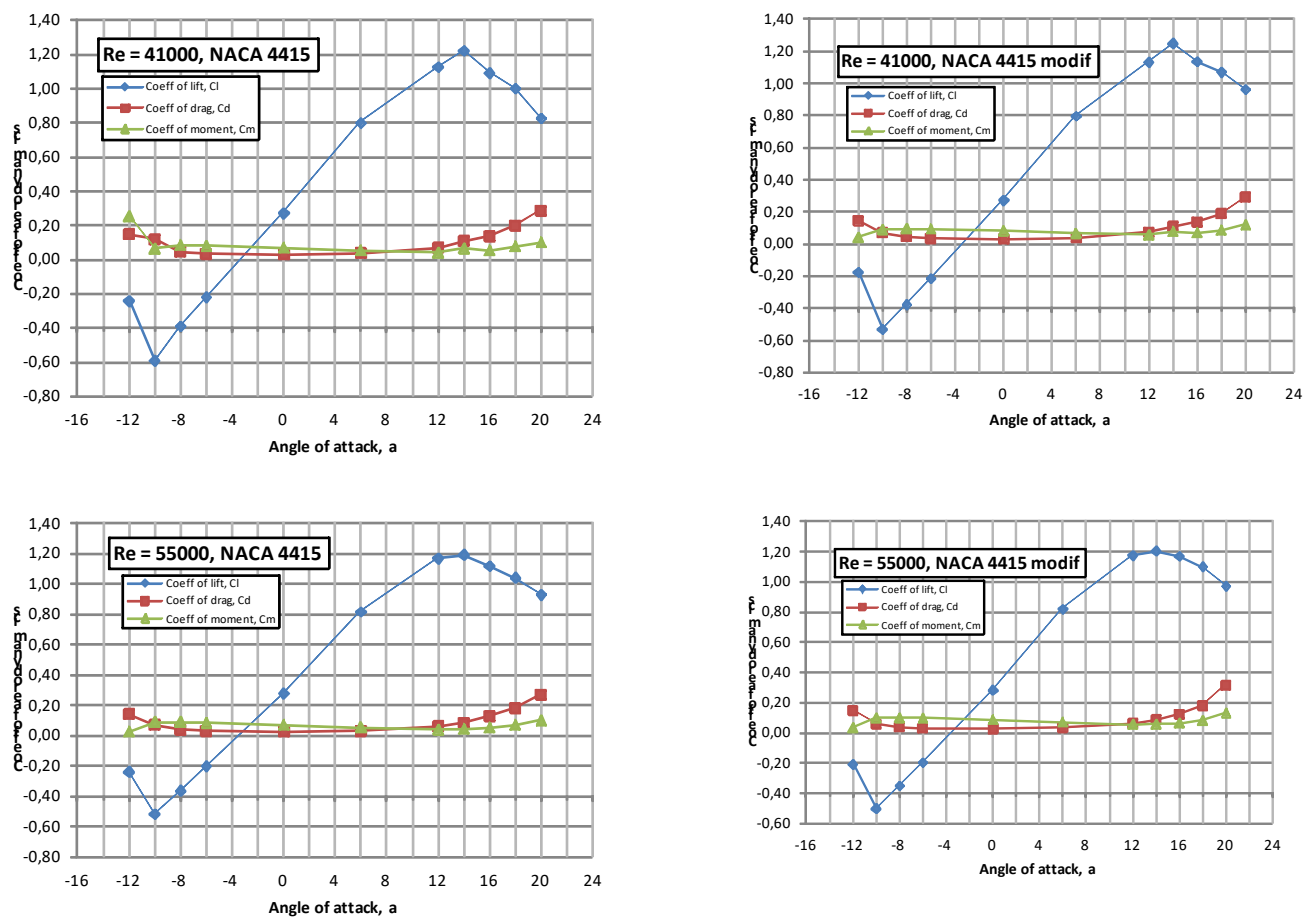

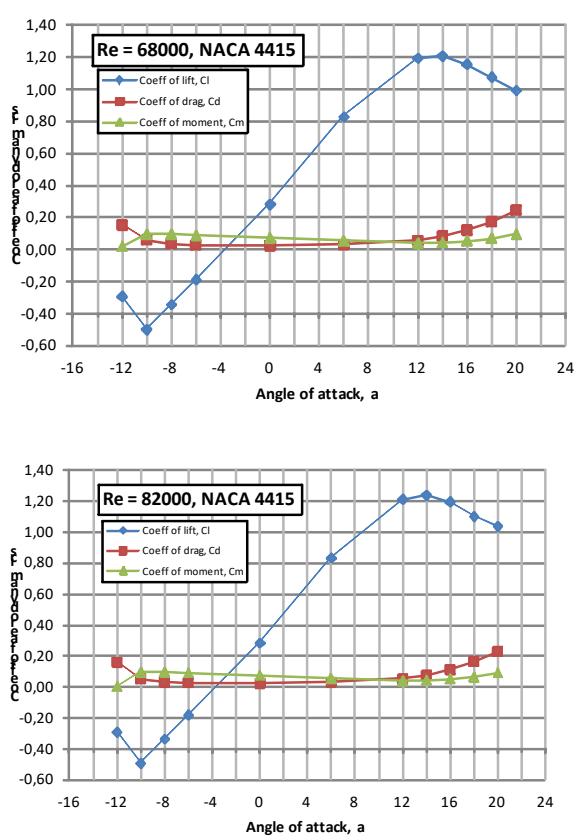
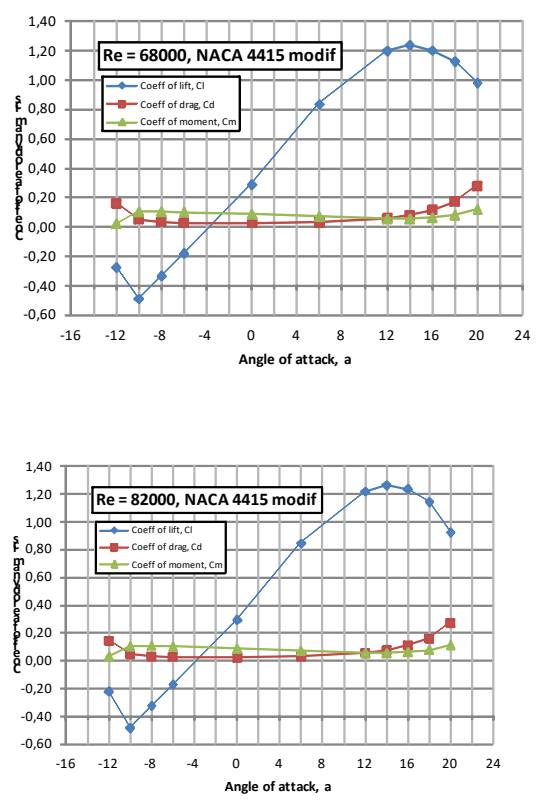

Fig. 6. Coefficient of aerodynamic of airfoil NACA 4415 and NACA 4415 modified

Further, the coefficient of lift (cl) coefficient of drag (cd) and coefficient of momentum (cm) are compared in Fig. 7 to Fig. 9. The value of lift coefficient of modified NACA 4415 is relatively higher than NACA 4415 , especially at $-12^{\circ}<\alpha<-8^{\circ}$ and $12^{\circ}<\alpha<20^{\circ}$. The drag coefficient of the modified NACA 4415 values are relatively similar with NACA 4415 especially at $-8^{\circ}<\alpha<16^{\circ}$. Whereas, the momentum coefficient of the modified NACA 4415 is relatively greater than the NACA 4415 at $-12^{\circ}<\alpha<20^{\circ}$.
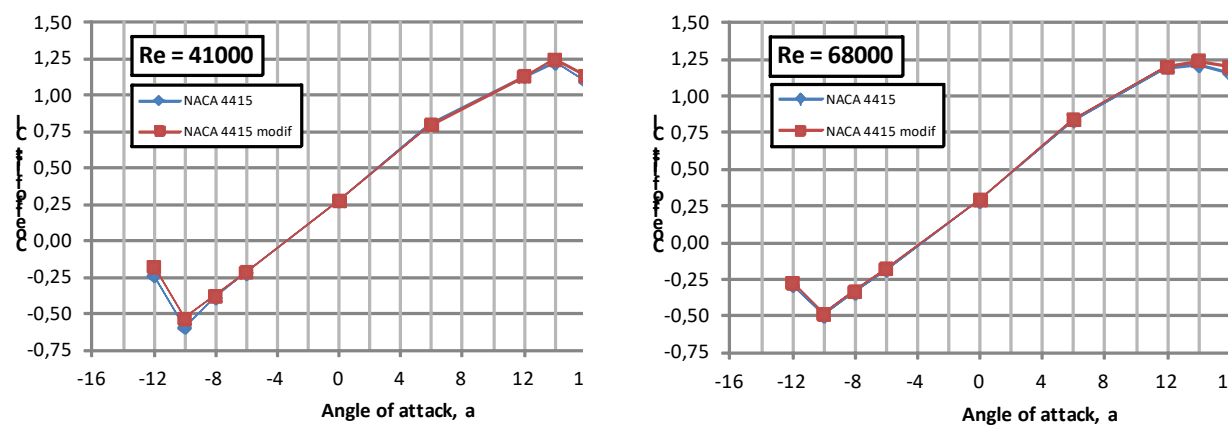

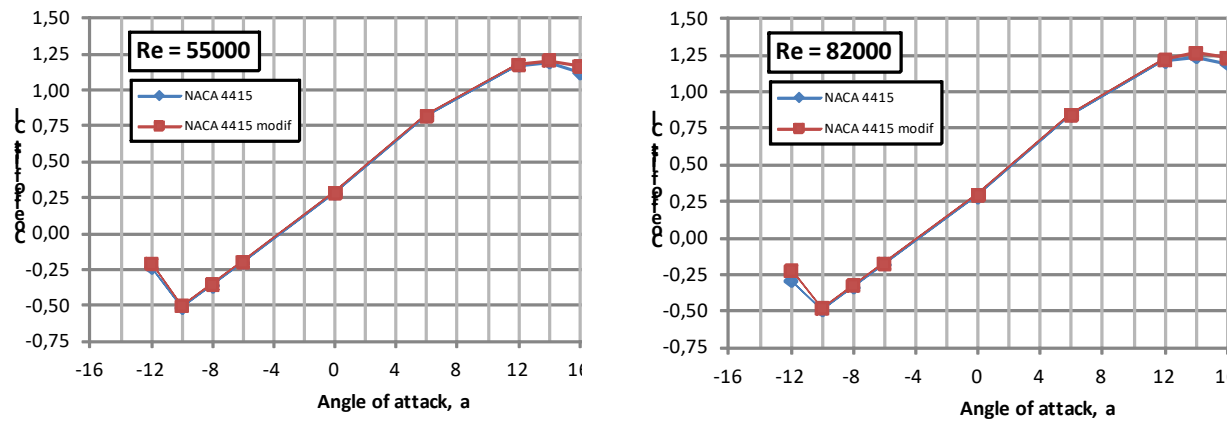

Fig. 7. The coefficient of lift (cl) of airfoils NACA 4415 and modified NACA 4415
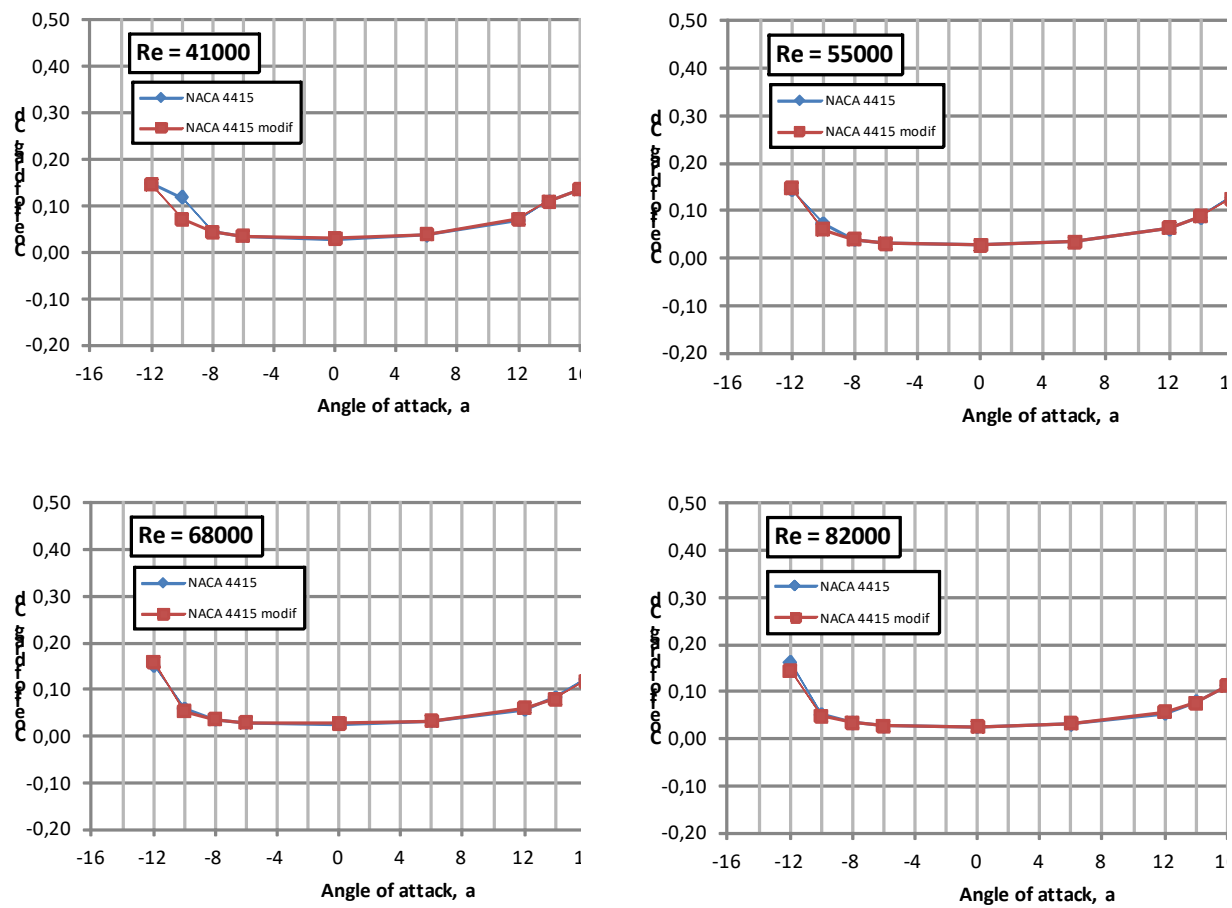

Fig. 8. The coefficient of drag (cd) of airfoils NACA 4415 and modified NACA 4415 



Fig. 9. The coefficient of momentum $\left(\mathrm{c}_{\mathrm{m}}\right)$ of airfoils NACA 4415 and modified NACA 4415

The performance the airfoils can be determined by their polar drag curve that is the lift coefficient curve to the drag coefficient as shown in Fig.10. It can be seen that the modified NACA 4415 polar drag curve is relatively higher than the NACA 4415 polar drag curve. Based polar drag curves, the modified NACA 4415 airfoil can be used as a basis for the formation of wind turbine propellers that can be twisted as far as a maximum of $20^{\circ}$ at the root (base) of wind turbine propellers and $0^{\circ}$ in the tip of wind turbine propellers.

From the results of the CFD analysis, the propeller of the wind turbine in the present work is fabricated which its twist angle of $15^{\circ}$ at the root side and untwisted at the tip. The propeller is fabricated using the composite material of Albizia wood, ramie fiber and resin. The propeller and the cross sectional area of the propeller are displayed in Fig. 11.
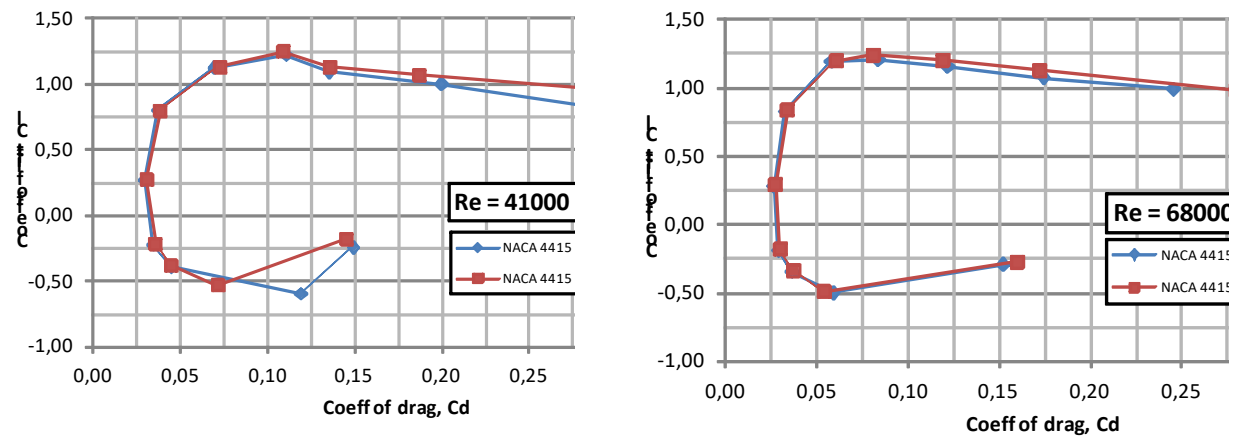

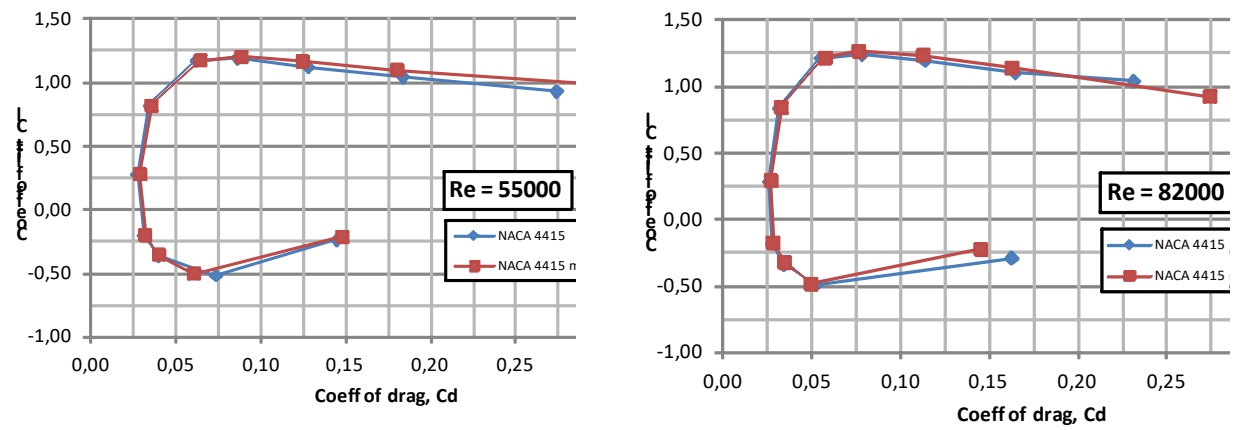

Fig.10. The polar drag of airfoils NACA 4415 and modified NACA 4415
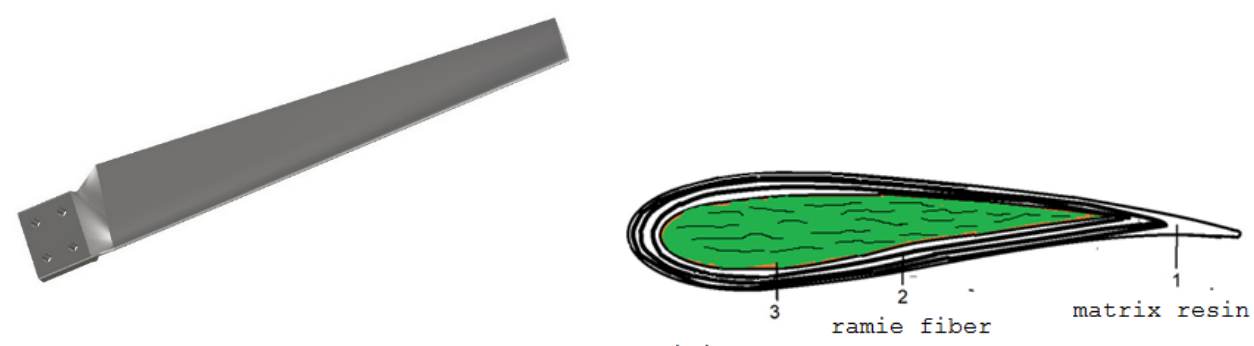

albisia falcata

Fig. 11. The propeller of the wind turbine with modified NACA 4415 airfoil

\section{Conclusion}

It can be concluded that the Spallart Allmaras (S-A) turbulent model is proven model for predicting the aerodynamic model of airfoils NACA 4415 and modified NACA 4415. The modified NACA 4415 airfoil has better lift and momentum coefficient. The value of lift coefficient of modified NACA 4415 is relatively higher than NACA 4415 , especially at $-12^{\circ}$ $<\alpha<-8^{\circ}$ and $12^{\circ}<\alpha<20^{\circ}$. In addition, the momentum coefficient of the modified NACA 4415 is also higher than the NACA 4415 at $-12^{\circ}<\alpha<20^{\circ}$.

The authors would like to thank DRPM-Kementerian Riset dan Pendidikan Tinggi for providing the financial to conduct this research. 


\section{References}

[1] Dirjen ESDM, Indonesian Outlook Energy 2006-2025, Jakarta.

[2] L. Wang, R. Quant, A. Kolios, Fluid structure interaction modelling of horizontal-axis wind turbine blades based on CFD and FEA, J. Wind Eng. Ind. Aerodyn. 158, 11-25 (2016)

[3] M.M. Elsakka, D.B. Ingham, L. Ma, M. Pourkashanian, CFD analysis of the angle of attack for a vertical axis wind turbine blade, Energy Conversion and Management 182, 154-165 (2019)

[4] H. Rahimi, J.G. Schepers, W.Z. Shen, N. Ramos García, M.S. Schneider, D. Micallef, C.J. Simao Ferreira, E. Jost, L. Klein, I. Herraez, Evaluation of different methods for determining the angle of attack on wind turbine blades with CFD results under axial inflow conditions, Renewable Energy 125, 866-876 (2018)

[5] H. Lee and D-J. Lee, Numerical investigation of the aerodynamics and wake structures of horizontal axis wind turbines by using nonlinear vortex lattice method, Renewable Energy 132, 1121-1133 (2019)

[6] M. Abdelwaly, H. El-Batsh, M.B. Hanna, Numerical study for the flow field and power augmentation in a horizontal axis wind turbine, Sustainable Energy Technologies and Assessments 31, 245-253 (2019)

[7] A. Suresh and S. Rajakumar, Design of small horizontal axis wind turbine for low wind speed rural applications, Materials Today: Proceedings 23, 16-22 (2020)

[8] A. Boujleben, A. Ibrahimbegovic, E. Lefrançois, An efficient computational model for fluid structure interaction in application to large overall motion of wind turbine with flexible blades, Applied Mathematical Modelling 77, 392-407 (2020)

[9] C.J Bai, W.C. Wang, Review of computational and experimental approaches to analysis of aerodynamic performance in horizontal-axis wind turbines (HAWTs), Renewable and Sustainable Energy Reviews 63, 506-519 (2016)

[10] K. Oukassou, S. El Mouhsine, A. El Hajjaji, B. Kharbouch, Comparison of the power, lift and drag coefficients of wind turbine blade from aerodynamics characteristics of NACA0012 and NACA2412, Procedia Manufacturing 32, 983-990 (2019)

[11] H.Y. Xu, C.L. Qiao, H.Q. Yang, Z.Y.Ye, Delayed detached eddy simulation of the wind turbine airfoil S809 for angles of attack up to 90 degrees, Energy 118, 10901109 (2017) 
[12] B. Wen, X. Tian, X. Dong, Z. Peng, W. Zhang, K. Wei, A numerical study on the angle of attack to the blade of a horizontal-axis offshore floating wind turbine under static and dynamic yawed conditions, Energy 168, 1138-1156 (2019)

[13] M. Bakirci, S. Yilmaz, Theoretical and computational investigations of the optimal tip-speed ratio of horizontal-axis wind turbines, Engineering Science and Technology, an International Journal 21, 1128-1142 (2018)

[14] Sudarsono, A.A.P. Susastriawan, Sugianto, Three-dimensional cfd analysis of performance of small-scale hawt based on modified naca-4415 airfoil, International Journal of Technology 10, 212-221 (2019)

[15] www.FLUENT.com 